Recepción: 15 / 04 / 2017

Ciencias Administrativas

Aceptación: 01 / 05 / 2017

Articulo Científico

Publicación: 15 / 05 / 2017

\title{
Unidades de negocio como fuente de emprendimiento y su incidencia en las prácticas profesionales de estudiantes universitarios
}

Business units as a source of entrepreneurship and their impact on the professional practices of university students

As unidades de negócios como uma fonte de empreendedorismo e seu impacto sobre as práticas profissionais dos estudantes universitarios

Lorena I. Muñoz-Oviedo

lmunoz@utb.edu.ec

Mariella G. Parrales-Higuera" mparrales@utb.edu.ec

Doris G. Naranjo-Chávez "' dnaranjo@utb.edu.ec

Correspondencia: 1munoz@utb.edu.ec

Magister en Administración de Empresas; Diploma Superior en Docencia Universitaria; Ingeniera Comercial; Universidad Técnica de Babahoyo, Ecuador.

II. Magister en Administración de Empresas; Ingeniera Comercial; Universidad Técnica de Babahoyo, Ecuador.

III. Magister en Contabilidad y Auditoría; Contador Público Auditor; Licenciada en Sistemas Computacionales; Tecnóloga en Computación e Informática; Universidad Técnica de Babahoyo, Ecuador. 


\section{Resumen}

La presente tesis condensa en su contenido una clara visión de la problemática sobre el proceso de pasantías o prácticas profesionales de los estudiantes, los mismos que no la están realizando en sus respectivos perfiles profesionales, y que ocurre por no existir una oferta de empresas suficientes para cubrir la cantidad de estudiantes en el proceso de realizarlas.

Es de importancia que el egresado de una carrera profesional el tener una formación y desarrollo práctico en el área en la cual se va a desempeñar como un profesional, y que mejor que hacerlo en un escenario real, y; que a través de ello visualice una oportunidad de desarrollar mejor su aptitudes mentales relacionadas con su perfil.

Dentro de los principales problemas detallados en el documento de trabajo que aquí se presenta se pueden identificar: Que los estudiantes no se encuentran realizando sus pasantías o prácticas profesionales en su perfil, el tiempo para realizarlas suele prolongarse por no encontrarse un banco suficientes de empresas en las cuales realizar las pasantías o prácticas, no se realizan las pasantías con una proyección de obtener una visión de emprendimiento en la carrera a concluir, lo que resulta en que el profesional egresado no empiece un negocio sino busque empleo que en la gran mayoría resulta poco práctico por ser la oferta laboral muy escasa para la cantidad de profesionales egresados.

Palabras clave: Fuente de emprendimiento; estudiantes universitarios; profesional. 


\begin{abstract}
This thesis condenses in content a clear view of the problem on the process of internships or work experience for students, the same as not being made in their respective professional profiles, and happens not to be a sufficient supply companies to cover the amount of students in the process of conducting.
\end{abstract}

It is important that the graduate of a professional career have training and practical development in the area in which they will play like a professional, and what better to do on a real stage, and; that through this visualize a better opportunity to develop their mental skills related to their profile.

Among the main problems outlined in the working paper presented here can be identified: Students are not doing their internships or work experience in their profile, time to perform them usually lasts for not being one sufficient bank companies which perform internships or practical internships are not projecting get a glimpse of entrepreneurship in the race to conclude, resulting in the graduate professional not start a business but seeking employment in the vast majority is little practical for being the very low labor market in number of graduates professionals.

Key words: Source of enterprise; University students; professional. 


\section{Resumo}

Esta tese condensa em conteúdo uma visão clara do problema sobre o processo de estágio ou experiência de trabalho para os alunos, o mesmo que não sendo feito em seus respectivos perfis profissionais, e não é ser um suprimento suficiente para cobrir a quantidade de estudantes em $\mathrm{O}$ processo de condução.

É importante que os graduados de uma carreira profissional tenham formação e desenvolvimento prático na área em que eles vão jogar como um profissional, eo que melhor para fazer em um estágio real, e; Que através deste visualizar uma melhor oportunidade para desenvolver suas habilidades mentais relacionadas com o seu perfil.

Entre os principais problemas delineados no documento de trabalho aqui apresentado podem ser identificados: Os alunos não estão fazendo seus estágios ou experiência de trabalho em seu perfil, o tempo para realizá-los costuma não ser uma empresa bancária suficiente que realizam estágios ou estágios práticos não estão projetando Obter um vislumbre do empreendedorismo na corrida para concluir, resultando no profissional de pós-graduação não iniciar um negócio, mas que procuram emprego na grande maioria é pouco prático para ser o mercado de trabalho muito baixo em número de profissionais de graduados.

Palavras chave: Fonte do empreendedorismo; estudantes universitarios; profissional. 


\section{Introducción.}

Esta investigación presenta el análisis de la problemática que enfrentan los estudiantes en etapa de realizar sus pasantías o prácticas profesionales y la utilidad que representa para la creación de Unidades de Negocio como fuente de emprendimiento en la Facultad de Administración, Finanzas e Informática de la Universidad Técnica de Babahoyo. (Raposo Rivas \& Zabalza Beraza, 2011)

En el desarrollo del siguiente estudio se encontrará una descripción de como las Unidades de Negocio como fuente de emprendimiento pueden mejorar la experiencia de las pasantías o prácticas profesionales y encaminar al estudiante en el emprendimiento en su propio perfil profesional. (Cornejo Abarca, 2014)

La motivación principal para seleccionar este tema, está dada por la necesidad existente de tener estudiantes en proceso de pasantías o prácticas que no están realizando las mismas en sus perfiles profesionales, lo que produce que el estudiante no se encuentre en una condiciones de consolidar su perfil profesional y a su vez proyectarse en un negocio donde poder cumplir objetivos personales, profesionales que es la aspiración de todo profesional egresado, por tanto se presenta una propuesta ágil para generar una gestión oportuna y acorde a las necesidades que en la actualidad son de importancia en la educación en todos sus niveles, esto es formar personas con una perspectiva emprendedora con la cual se formen empresas que mejoren la calidad de vida y contribuyan a los objetivos nacionales que es cambiar la matriz productiva del país, que es formar el talento humano en lo técnico y científico, con proyección local, regional, nacional e internacional. (Pinnegar \& Hamilton, 2009) 
Esta investigación está basada en la importancia que tiene que los estudiantes adquieran una experiencia basada en sus perfiles profesionales que los ubiquen en mercado laboral como generadores de emprendimiento a un mediano y largo plazo como empresarios creadores de riqueza, por lo tanto es de suma importancia tener todo el apoyo de parte administrativa, siendo favorable el levantamiento de información desde el punto de vista de los involucrados: Decano, Subdecano estudiantes, Directores de la Unidad de Servicios a la Comunidad, y demás personas involucradas en la educación superior, contribuyendo de esta forma a mejorar la calidad de las pasantías o prácticas profesionales encaminadas a consolidar el perfil profesional del egresado. (Sisto, 2011) (Reyes, Cornejo, Arévalo, \& Sánchez, 2010)

El objetivo del presente estudio fue analizar el impacto que tendrá la creación de unidades de negocios como fuente de emprendimiento para los estudiantes de la Universidad Técnica de Babahoyo, F.A.F.I que realicen pasantías o prácticas profesionales.

\section{Metodología.}

La razón de elaborar este trabajo de investigación es de considerar y dar a conocer la importancia que tiene el que un egresado de una carrera profesional tenga una formación y desarrollo práctico en el área en la cual se va a desempeñar como un profesional, y que mejor que hacerlo en un escenario real, y; que a través de ello visualice una oportunidad de desarrollar mejor su aptitudes y competencias relacionadas con su perfil.

El comportamiento del emprendedor responde a una interacción directa con su entorno, y al constante esfuerzo por mantener la supervivencia de la especie humana, que le permite guiar la orientación cognoscitiva y generación de ideas a la satisfacción de necesidades básicas que evolucionan junto con el individuo, dando generando la creación de empresas e iniciativas 
productivas de todo tipo, siendo esto parte del proceso natural de la vida del hombre en toda su historia.

El interés de las Universidades por fomentar el "espíritu emprendedor" de sus estudiantes aumenta de manera directamente proporcional a los problemas de la tasa de desempleo, la deprivación y la pauperización de la economía global.

En todo el mundo, hay una explosión de Sesiones Picth, concursos de planes de negocios, maratones de creatividad empresarial, (con pocas variantes verdaderamente innovadoras) cursos de formación de emprendedores y actividades para fomentar el espíritu empresarial.

Las inciertas salidas ocupacionales de las carreras universitarias —aún de las más modernas como las de diseño, o las nuevas ramas de la ingeniería y la informática- impulsan a los estudiantes a plantearse si su destino depende más de lo que hagan por sí mismos que de un diploma que les otorga su Universidad.

Ante el surgimiento de un nuevo perfil de estudiante: "serás emprendedor por las buenas o por las malas". Vamos a denominarlos (sin rigor que "así serán”) “e-universitarios” (emprendedores universitarios) que no necesariamente es un concepto asociado al de Universidades Emprendedoras.

Las Universidades Emprendedoras pueden tener más base en su intención política para ser consideradas como tales, que por su verdadera vocación por lograrlo. En cambio, los "euniversitarios" tienen una intención o propósito empresarial diferente al de crear "un negocio".

Los e-universitarios no solo se centran en emprender un negocio rentable, sino en la construcción de un negocio sustentable en el tiempo y conectado con el progreso general de su comunidad; incluso, es frecuente que este nuevo perfil de emprendedores de origen académico estén 
inspirados por un propósito sin fines de lucro y una visión filantrópica de su propio ejercicio profesional en función de la calidad de vida de su sociedad.

Es más fácil para nuestra idiosincrasia latina decir lo que "no es" y señalar "lo que no debe hacer" un centro o programa para el desarrollo emprendedor en el ámbito universitario (esperanzadamente, así podremos entender mejor el lado proactivo de la cuestión: qué ser y cómo hacer).

No todas las universidades pueden crear, desarrollar y gestionar algo como la Fundación Kauffman, ni todas las universidades tienen las capacidades, los recursos físicos y talentos humanos como la Universidad de Georgetown, o la Escuela Babson.

Lo más importante es evitar suponer que se puede tener "uno de esos" porque se han leído un par libros sobre la metodología de la formación emprendedora en la Universidad de Stanford.

\section{Resultados.}

Pruebas estadísticas aplicadas en la verificación de la hipótesis.

Para la verificación de la hipótesis de este proceso de investigación se ha realizado la aplicación de los instrumentos de investigación y luego se ha detallado la información relevante utilizando el sistema operativo Excel, a través del cual se aplicó la frecuencia relativa a fin de determinar cuáles son los aspectos relevantes de los datos obtenidos.

Análisis e interpretación de datos

Procesamiento del primer instrumento de recolección de la información: Encuesta aplicada a los estudiantes de la FAFI. 
¿Conoce usted de la existencia de unidades de negocios en la Universidad Técnica de Babahoyo?

\section{Cuadro $\mathrm{N}^{\circ}$ 1: Unidades de negocios.}

\begin{tabular}{|c|c|c|}
\hline OPINIÓN & ENCUESTADOS & PORCENTAJE (\%) \\
\hline $\mathrm{Si}$ & 33 & $17 \%$ \\
\hline No & 162 & $83 \%$ \\
\hline TOTAL & $\mathbf{1 9 5}$ & $\mathbf{1 0 0 \%}$ \\
\hline
\end{tabular}

En consideración al Cuadro $\mathrm{N}^{\circ} 1$ un $17 \%$ de los estudiantes conoce de la existencia de Unidades de negocios en la UTB, mientras que un $83 \%$ no conoce de las existencias de las mismas.

\section{¿Cómo calificaría usted sus pasantías?}

\section{Cuadro $\mathrm{N}^{\circ}$ 2: Calificación de pasantías o prácticas pre-profesionales}

\begin{tabular}{|c|c|c|}
\hline OPINIÓN & ENCUESTADOS & PORCENTAJE (\%) \\
\hline Excelente & 12 & $6 \%$ \\
\hline Muy Buena & 23 & $12 \%$ \\
\hline Buena & 33 & $17 \%$ \\
\hline Regular & 51 & $26 \%$ \\
\hline Susceptible de mejora & 76 & $39 \%$ \\
\hline TOTAL & $\mathbf{1 9 5}$ & $\mathbf{1 0 0} \%$ \\
\hline
\end{tabular}

De acuerdo al Cuadro $\mathrm{N}^{\circ} 2$ la gran mayoría de los estudiantes califica sus pasantías como regulares y susceptibles de mejora, lo que indica que hay que implementar un mejor sistema para que el estudiante realice sus pasantías o prácticas pre profesionales. Este sistema a implementar debe 
de estar de acuerdo con las exigencias del tiempo en que se actúa estas son las de crear una cultura emprendedora para el surgimiento de empresas.

¿De tener la oportunidad de realizar preparaciones pre-profesionales en una empresa le agradaría que estén de acuerdo a sus competencias profesionales en formación?

\section{Cuadro $\mathbf{N}^{\circ}$ 3: Competencias profesionales.}

\begin{tabular}{|c|c|c|}
\hline OPINIÓN & ENCUESTADOS & PORCENTAJE (\%) \\
\hline $\mathrm{Si}$ & 195 & $100 \%$ \\
\hline No & 0 & $0 \%$ \\
\hline TOTAL & $\mathbf{1 9 5}$ & $\mathbf{1 0 0} \%$ \\
\hline
\end{tabular}

La Cuadro $\mathrm{N}^{\circ} 3$ indudablemente muestra que los estudiantes adquieren un perfil profesional de acuerdo a su carrera, por lo tanto ellos desean que sus prácticas pre-profesionales o pasantías sean de acuerdo a éste perfil. El 100\% de los estudiantes de tener la oportunidad de realizar las practicas pre profesionales las realizarían de acuerdo a las competencias profesionales adquiridas en sus respectivas formación.

¿Se siente Ud. afectado en el proceso de pasantías o prácticas profesionales al no existir una empresa donde realizarlas? 


\section{Cuadro $\mathbf{N}^{\circ}$ 4: Proceso de pasantías.}

\begin{tabular}{|c|c|c|}
\hline OPINIÓN & ENCUESTADOS & PORCENTAJE (\%) \\
\hline Muy Afectado & 107 & $55 \%$ \\
\hline Afectado & 56 & $29 \%$ \\
\hline Poco Afectado & 23 & $12 \%$ \\
\hline Nada Afectado & 9 & $4 \%$ \\
\hline TOTAL & $\mathbf{1 9 5}$ & $\mathbf{1 0 0} \%$ \\
\hline
\end{tabular}

En consecuencia la Cuadro $\mathbf{N}^{\circ} 4$ resultado es evidente de acuerdo a la, los estudiantes se sienten afectados y muy afectados al no existir empresas donde puedan realizar las prácticas, que refuerzan lo aprendido en sus estudios; y, que las mismas le brinden una oportunidad laboral por cuenta propia o por medio de empleo.

El expresa que los estudiantes en un 55\%se sienten muy afectados, un $29 \%$ afectado, un $12 \%$ poco afectado, y un $4 \%$ nada afectado, lo que demuestra que una gran mayoría en etapa de este proceso necesita que este proceso mejore.

¿Debería la Unidad de Servicios a la Comunidad disponer de nuevas opciones para realizar las prácticas o pasantías pre-profesionales? 


\section{Cuadro $\mathbf{N}^{\circ}$ 5: Opciones de pasantías o prácticas pre-profesionales.}

\begin{tabular}{|c|c|c|}
\hline OPINIÓN & ENCUESTADOS & PORCENTAJE (\%) \\
\hline $\mathrm{Si}$ & 195 & $100 \%$ \\
\hline $\mathrm{No}$ & 0 & $0 \%$ \\
\hline TOTAL & $\mathbf{1 9 5}$ & $\mathbf{1 0 0 \%}$ \\
\hline
\end{tabular}

En consideración la Cuadro $\mathrm{N}^{\circ} 5$ la USC es una ayuda invaluable en lo que tiene que ver con el enlace entre la comunidad y la comunidad siendo su muestra de ello los mismo lo que expresa la estudiantes y su aporte de conocimiento, por lo tanto es evidente que está USC brinden una gran variedad de opciones en lo que respecta a realizar las prácticas o pasantías preprofesionales a los estudiantes de acuerdo a sus perfiles profesionales.

Un $100 \%$ los estudiantes están de acuerdo en que la USC debe de disponer de nuevas opciones para realizar las prácticas o pasantías pre-profesionales. Lo que da una alerta para que ésta unidad realice un estudio de opciones actuales y las que a futuro se puedan implementar.

\section{¿Cómo califica Ud. la oferta de empresas para que realicen pasantías o prácticas pre- profesionales los estudiantes en dicho proceso?}




\section{Cuadro $\mathbf{N}^{\circ}$ 6: Ofertas de empresas.}

\begin{tabular}{|c|c|c|}
\hline OPINIÓN & ENCUESTADOS & PORCENTAJE (\%) \\
\hline Suficiente & 30 & $15 \%$ \\
\hline Insuficiente & 110 & $57 \%$ \\
\hline Susceptible de incremento & 55 & $28 \%$ \\
\hline TOTAL & $\mathbf{1 9 5}$ & $\mathbf{1 0 0} \%$ \\
\hline
\end{tabular}

De acuerdo al Cuadro $\mathrm{N}^{\circ} 6$ los estudiantes consideran que la actual oferta de empresas es deficiente donde poder realizar pasantías o prácticas pre-profesionales, nos demuestra que en un 57\% los estudiantes califican que existe una insuficiente oferta de empresas donde se pueda realizar las pasantías o practicas pre-profesionales.

Lo que amerita gestionar de forma urgente un sistema que agilice el sistema para que los estudiantes puedan realizar sus prácticas en forma eficiente y en sus perfiles profesionales.

¿Considera Ud. que se reduciría el tiempo en el proceso pasantías o prácticas profesionales si se contará con más lugares donde realizarlas?

\section{Cuadro $\mathrm{N}^{\circ}$ 7: Tiempo en el proceso de pasantías.}

\begin{tabular}{|l|l|l|}
\hline OPINIÓN & ENCUESTADOS & PORCENTAJE (\%) \\
\hline Considerablemente & 65 & $33 \%$ \\
\hline Notablemente & 130 & $67 \%$ \\
\hline Discretamente & 0 & $0 \%$ \\
\hline TOTAL & $\mathbf{1 9 5}$ & $\mathbf{1 0 0} \%$ \\
\hline
\end{tabular}


Por lo expresado en la Cuadro $\mathrm{N}^{\circ} 7$ los estudiantes al estar en sus últimos semestres consideran de importancia incorporarse a la actividad laboral, por lo tanto el tiempo es de mucha importancia al momento de asignarles ya sea las pasantías o prácticas profesionales, para agilizar su trámite para egresar y por ende los de graduación. Se considera que en un $67 \%$ se reduciría el tiempo en el proceso de pasantías o prácticas pre-profesionales si se contara con más lugares donde realizarlas, así no tendrían que esperar que un grupo las realizara y así continuar los que están en espera.

\section{¿Cree Ud. que las pasantías deberían ser oportunidades para empezar su vida profesional dentro de su perfil?}

\section{Cuadro $\mathrm{N}^{\circ}$ 8: Oportunidades dentro del perfil.}

\begin{tabular}{|c|c|c|}
\hline OPINIÓN & ENCUESTADOS & PORCENTAJE (\%) \\
\hline $\mathrm{Si}$ & 195 & $100 \%$ \\
\hline $\mathrm{No}$ & 0 & $0 \%$ \\
\hline TOTAL & $\mathbf{1 9 5}$ & $\mathbf{1 0 0} \%$ \\
\hline
\end{tabular}

En los datos del Cuadro $\mathrm{N}^{\circ}$ 8, al ser las pasantías un entrenamiento para lo que será la vida profesional, los estudiantes desean que estas sean lo más cercano a la realidad de sus vidas como profesional en sus respectivos perfiles. En su totalidad el cuadro muestra que las pasantías son una oportunidad de comenzar la vida profesional dentro del perfil. 
¿Considera Ud. el emprendimiento una fuente para mejorar las prácticas pre-profesionales o pasantías de la F.A.F.I?

Cuadro $\mathbf{N}^{\circ}$ 9: Emprendimiento como fuente de prácticas pre-profesionales.

\begin{tabular}{|c|c|c|}
\hline OPINIÓN & ENCUESTADOS & PORCENTAJE (\%) \\
\hline $\mathrm{Si}$ & 132 & $68 \%$ \\
\hline $\mathrm{No}$ & 63 & $32 \%$ \\
\hline TOTAL & $\mathbf{1 9 5}$ & $\mathbf{1 0 0} \%$ \\
\hline
\end{tabular}

Lo que demuestra el Cuadro $\mathrm{N}^{\circ} 9$ actualmente el emprendimiento se considera una fuente de poner en práctica todo el potencial que se adquiere a través de competencias propias de una carrera o profesión siendo éste la mejor forma de iniciarse profesionalmente, de ahí que el emprendimiento se considere una muy buena alternativa. Nos indica por sus resultados que en un $68 \%$ los estudiantes consideran como una opción de mejora al emprendimiento, siendo éste una forma de empezar de forma directa su vida profesional y de superación personal y económica.

De crearse unidades de negocios como opción donde realizar las prácticas profesionales o pasantías, las consideraría. 


\section{Cuadro $\mathbf{N}^{\circ}$ 10: Las unidades de negocio como opción rentable.}

\begin{tabular}{|c|c|c|}
\hline OPINIÓN & ENCUESTADOS & PORCENTAJE (\%) \\
\hline Rentable & 118 & $61 \%$ \\
\hline Poco rentable & 51 & $26 \%$ \\
\hline No rentable & 26 & $13 \%$ \\
\hline TOTAL & $\mathbf{1 9 5}$ & $\mathbf{1 0 0} \%$ \\
\hline
\end{tabular}

En consideración a lo expresado en el Cuadro $\mathrm{N}^{\circ} 10$ la creación de unidades de negocio se consideraría como una buena opción para realizar las prácticas profesionales o pasantías, de parte de los estudiantes ya que les daría la opción de hacer las prácticas en sus respectivos perfiles y mejorar la visión de verse como un emprendedor desarrollando sus competencias profesionales. Nos demuestra que en un $61 \%$ considera que es rentable la opción de realizar las prácticas profesionales o pasantías, considerando la posibilidad de emprender un negocio que se alinee con su perfil profesional. 


\section{Procesamiento del Segundo instrumento de recolección de la información.}

\section{Entrevistas realizadas: Decano, Subdecano, Jefe de Departamento de Vinculación la Sociedad}

\begin{tabular}{|c|c|}
\hline PREGUNTAS & ASPECTOS RELEVANTES \\
\hline $\begin{array}{l}\text { 1. ¿Qué impacto considera Ud. que tendría } \\
\text { la creación de unidades de negocios en la UTB y } \\
\text { en especial en la FAFI? }\end{array}$ & $\begin{array}{l}\text { Disminución de congestionamiento de las los } \\
\text { departamentos de vinculación, y el surgimiento } \\
\text { de nuevos profesionales capacitados dentro de } \\
\text { sus perfiles }\end{array}$ \\
\hline $\begin{array}{l}\text { Considera Ud. que se resolvería la } \\
\text { situación de ubicar a los estudiantes en sus } \\
\text { respectivos perfiles al crear unidades de } \\
\text { negocios como fuente de emprendimiento? }\end{array}$ & $\begin{array}{l}\text { Se resolvería, en la medida que el estudiante ve } \\
\text { realizadas la visión de tener una profesión de } \\
\text { acorde a los estudios realizados y que sea un } \\
\text { proyecto de vida que sirva para alcanzar metas } \\
\text { personales, profesionales y económicas }\end{array}$ \\
\hline $\begin{array}{l}\text { 3. Cómo cree Ud. que se reduciría en } \\
\text { tiempo de espera a los estudiantes al crear } \\
\text { unidades de negocios como fuente de } \\
\text { emprendimiento? }\end{array}$ & $\begin{array}{l}\text { El tiempo es un factor importante a considerar al } \\
\text { momento de terminar los estudios, representando } \\
\text { un incremento en los presupuestos cuando no se } \\
\text { dan dentro del tiempo planificado. Esto } \\
\text { contribuiría a mejorar la eficacia en términos de } \\
\text { lograr las metas trazadas tanto por las } \\
\text { autoridades como estudiantes. }\end{array}$ \\
\hline $\begin{array}{l}\text { 4. Cree Ud. que es necesario aumentar el } \\
\text { emprendimiento para lograr que los estudiantes } \\
\text { puedan iniciarse en empresas que estén de }\end{array}$ & $\begin{array}{l}\text { El emprendimiento es la base para mejorar la } \\
\text { economía, lo que se refleja en la creación de } \\
\text { nuevos emprendedores que llegaran a ser }\end{array}$ \\
\hline
\end{tabular}




\begin{tabular}{|c|c|}
\hline acuerdo a sus perfiles de egresados? & $\begin{array}{l}\text { empresarios que mejoraran los el entorno } \\
\text { económico de su comunidad y por ende la } \\
\text { calidad de vida. }\end{array}$ \\
\hline $\begin{array}{l}\text { 5. Cuál cree Ud. que sería una opción } \\
\text { viable para que los estudiantes mejoren su } \\
\text { experiencia en las pasantías prácticas pre } \\
\text { profesionales }\end{array}$ & $\begin{array}{l}\text { Lo importante para mejorar la experiencia es que } \\
\text { el estudiante realice sus pasantías o practicas pre } \\
\text { profesionales en su respectivos perfiles ya que } \\
\text { así se encontrará en una experiencia real de lo } \\
\text { que es o será su vida profesional en la carrera } \\
\text { escogida }\end{array}$ \\
\hline $\begin{array}{l}\text { 6. Cuál sería su propuesta como autoridad } \\
\text { en cuento a la creación de unidades de negocios? }\end{array}$ & $\begin{array}{l}\text { La propuesta es de apoyar aquella alternativa de } \\
\text { la creación de unidades de negocio como fuente } \\
\text { de emprendimiento, y que se contribuya a } \\
\text { mejorar la calidad de los estudiantes egresado y } \\
\text { su perfil profesional }\end{array}$ \\
\hline $\begin{array}{l}\text { 7. De presentarse un proyecto para } \\
\text { implementarlo como una solución viable que } \\
\text { contribuya a mejorar la calidad del perfil del } \\
\text { egresado a través de la creación de unidades de } \\
\text { negocio lo apoyaría? }\end{array}$ & $\begin{array}{l}\text { El apoyo es incondicional, la Universidad está } \\
\text { contribuyendo a mejorar la calidad de vida de la } \\
\text { comunidad y apoyando el surgimiento de nuevas } \\
\text { empresas. }\end{array}$ \\
\hline Cuál sería su aporte como autoridad para & El aporte sería el brindar la capacitación y \\
\hline
\end{tabular}




\begin{tabular}{|c|c|}
\hline $\begin{array}{l}\text { que estas unidades de negocio contribuyan a } \\
\text { solucionar el que los estudiantes realizan sus } \\
\text { prácticas pre profesionales o pasantías en sus } \\
\text { respectivos perfiles? }\end{array}$ & $\begin{array}{l}\text { desarrollo necesarios para que estas unidades de } \\
\text { negocio se conviertan en una fuente de constante } \\
\text { innovación productiva y que el futuro } \\
\text { profesional cree en su mente la visión de ser un } \\
\text { emprendedor y empresario }\end{array}$ \\
\hline $\begin{array}{l}\text { 9. Qué opinión tiene Ud. respecto a mejorar } \\
\text { los perfiles de los estudiantes y la creación de } \\
\text { micro emprendimiento que contribuiría las } \\
\text { unidades de negocios? }\end{array}$ & $\begin{array}{l}\text { La opiniones que se tienen al respecto es que sí } \\
\text { se contribuye a través de la creación de unidades } \\
\text { de negocios a crear de negocios encaminados al } \\
\text { micro emprendimiento y estos a su vez a mejorar } \\
\text { el perfil del estudiante, gana tanto la Universidad } \\
\text { como el profesional que está egresando y la } \\
\text { calidad de su educación se eleva y esto sirve de } \\
\text { reconocimiento de la labor en la educación de } \\
\text { superior. }\end{array}$ \\
\hline $\begin{array}{l}\text { 10. Cree Ud. que mejorando la calidad del } \\
\text { perfil del egresado a través de implementar las } \\
\text { unidades de negocio como fuente de } \\
\text { emprendimiento se mejoraría la calidad del } \\
\text { perfil del egresado? }\end{array}$ & $\begin{array}{l}\text { Sin lugar a dudas, es un esfuerzo que vale la } \\
\text { pena implementar, lo que se quiere es preparar } \\
\text { profesionales que puedan realizarse en sus } \\
\text { respectivas actividades profesionales y } \\
\text { económicas haciéndolos miembros productivos } \\
\text { de la comunidad. }\end{array}$ \\
\hline
\end{tabular}


La creación de las unidades de negocio tiene una admirable aceptación según muestra el cuadro, la importancia que encierra que se tenga el proceso y se convierta en un sistema que permita a estudiantes medio de desarrollar sus capacidades, habilidades y destrezas que se convierten en competencias profesionales junto a resultados de su trabajo, sus experiencias convertidas en emprendimientos propios de sus perfiles profesionales y el mérito que lleva implícito el centro de educación, la Universidad Técnica de Babahoyo.

\section{Conclusiones.}

La creación de unidades de negocios en la Universidad Técnica de Babahoyo tiene una aceptación por parte de estudiantes y autoridades por ser este un medio por el cual se llegaría a mejorar la afectación que tienen los estudiantes en realizar sus pasantías o practicas pre profesionales por no encontrar en la ciudad ni en la provincia una oferta por parte de empresas que cubra la demanda de estudiantes en este proceso, prolongando el tiempo para realizarlas. Se hace urgente por lo tanto la implementación de un nuevo sistema que permita que los estudiantes realicen las pasantías o practicas pre profesionales en sus respectivos perfiles y que obtengan a través de éstas una experiencia real de lo que se viene en su vida profesional, y; cómo contribuirá su desempeño en su carrera a mejorar su economía en lo personal, familiar y en la comunidad.

El impacto que tiene el que no se disponga de un banco suficiente de empresas, disminuye su impacto al tener como una opción las unidades de negocios como fuente de emprendimiento, estas llevarían a agilizar la ubicación de estudiantes en procesos de pasantías.

Al disminuir la problemática de tener estudiantes rezagados en realizar sus pasantías, se contribuirá a tener una gestión más ágil en las Unidades de Servicio a la Comunidad, con suficientes 
planes de acción que permitan contribuir a mejorar la calidad del perfil profesional, logrando así una inserción real del profesional a su vida laboral.

La acogida que tiene la creación de unidades de negocio como propuesta, por parte de los estudiantes y autoridades muestra que estas unidades de negocios pueden contribuir en forma eficiente a las prácticas pre profesionales de estudiantes en sus respectivos perfiles y prepararlos para ser profesionales de éxitos en las áreas que realizaron sus estudios.

\section{Bibliografía.}

Cornejo Abarca, J. (2014). Prácticas profesionales durante la formación inicial docente: análisis y optimización de sus aportes a los que aprenden y a los que enseñan a aprender "a enseñar". Estudios Pedagógicos, 60, 239-256.

Pinnegar, S., \& Hamilton, M. (2009). Self-study of practice as a genre of Qualitative research. Dordrecht. Springer Series: The Nederlands.

Raposo Rivas , M., \& Zabalza Beraza, M. (2011). La formación práctica de estudiantes universitarios: repensando el Practicum. Revista de Educación, 354(1).

Reyes, L., Cornejo, R., Arévalo, A., \& Sánchez, R. (2010). Ser docente y subjetividad histórica en el Chile actual: discursos, práctica y resistencias. Polis, 9(27), 269-292.

Sisto, V. (2011). Nuevo profesionalismo y profesores: una reflexión a partir del análisis de las actuales políticas de profesionalización para la educación en Chile. Signo y Pensamiento, 31(59), 178-192. 